\title{
Trastornos de la conducta alimentaria no especificados, síndromes parciales y cuadros subclínicos: U na alerta para la atención primaria
}

\author{
Rosa Behar A.
}

\section{Eating disorders not otherwise specified, partial syndromes and subclinical disorders: A warning in primary care}

Eating disorders not otherwise specified are the most common category of eating disorders encountered in routine clinical practice. Their prevalence fluctuates from $2.4 \%$ to $12.6 \%$ among female adolescents and up to $60 \%$ of cases in treatment centres. Binge eating disorder is the most differentiated subtype. Peripuberal period, diabetes mellitus type I, intense and increased physical activity, demographic and psychosociocultural aspects, physical and psychopathological diseases are risk factors. Clinical features, course, outcome and therapeutic approaches are similar to those of full syndromes. From an etiological point of view, some authors believe that there is a continuum from altered eating behaviors and concerns about body shape and weight to typical anorectic and/or bulimic disorders. Others classify them as subcategories of full or mixed disorders. In conclusion, these conditions are heterogeneous syndromes that need to be reconsidered from both nosological and conceptual perspectives. They also demand an early recognition and treatment and further research about these disorders is required (Rev Méd Chile 2008; 136: 1589-98).

(Key words: Adolescent; Anorexia nervosa; Bulimia nervosa; Eating disorders)

Recibido el 10 de enero, 2008. Aceptado el 8 de mayo, 2008.

Departamento de Psiquiatría, Universidad de Valparaíso, Chile.

\begin{abstract}
$\mathrm{A}$ unque la definición nosológica de los trastornos alimentarios ha sido optimizada en el tiempo, aún existen considerables dificultades en su clasificación ${ }^{1}$. Desde aproximadamente las dos últimas décadas surgen en la literatura especializada los así llamados síndromes parciales, subclínicos, subumbrales o atípicos de los trastornos
\end{abstract}

Correspondencia a: Dra. Rosa Behar A. Departamento de Psiquiatría, Universidad de Valparaíso. Valparaíso, Chile. Casilla 92-V, Valparaíso. Fono/Fax: (56) (32) 2508550.

E mail: rositabehar@vtr.net alimentarios, que incluyen formas de nutrición y alteraciones en la imagen corporal que no se ajustan claramente a las categorías más conocidas -anorexia nerviosa y bulimia nerviosa-, que dificultan la capacidad de vivir una vida óptima, interfiriendo con aspectos integrales de salud, trabajo, rendimiento académico o laboral o relaciones interpersonales. Las conductas pueden consistir en métodos de regulación ponderal, tales como ejercicios drásticos, dieta crónica, abuso de anorexígenos, laxantes, enemas o diuréticos, masticación y emisión del alimento sin ingerirlo y comilonas con menos frecuencia que en el trastorno por atracones. 
Trastornos DE LA CONDUCTA ALIMENTARIA NO ESPECIFICADOS

Definición y clasificación. El DSM-IV-TR (Manual Diagnóstico y Estadístico de los Trastornos Mentales. Texto Revisado) ${ }^{2}$ (Tablas 1 y 2 ) y la ICD-10 (Clasificación Internacional de Trastornos Mentales) ${ }^{3}$ (Tablas 3-7) han introducido estos síndromes en su nomenclatura como la categoría de los trastornos de la conducta alimentaria no especificados, conceptualmente confusa y que comprende un grupo clínicamente heterogéneo de diagnósticos ${ }^{4}$. Son condiciones catalogadas como residuales y tienden a ser desatendidas por los investigadores. Sin embargo, son los tipos de cuadros alimentarios más comunes en la práctica clínica rutinaria ${ }^{4}$ que ameritan una asistencia oportuna y una detección precoz, particularmente a nivel primario.

El DSM-IV-TR ${ }^{2}$ describe ilustraciones en las que se hace hincapié en la ausencia de criterios, pero sin proponer ninguna como trastorno específico o para su consideración futura. Comprende un gran número de pacientes con anorexia o bulimia nerviosas subsindromáticas que no poseen $85 \%$ bajo el peso esperado, o con menstruación regular o que presentan comilonas y purgas ligeramente en menor cantidad que dos veces por semana, como se requiere para la bulimia nerviosa completa. Además, se agregan pacientes con peso normal que purgan, individuos que mastican y escupen su alimento sin deglutirlo para prevenir la ganancia ponderal, y otros con trastornos por atracones 5 . También se incluyen a aquellos que experimentan discapacidad psiquiátrica debido al abuso de anorexígenos y diuréticos, otros que están obsesivamente preocupados con la liposucción como modalidad de manejo del peso y silueta y ciertos pacientes postgastrectomizados 6 . Otra posibilidad es que tan sólo se trate de síndromes transitorios o que anteceden en tiempo al establecimiento definitivo de un cuadro completo de anorexia o bulimia nerviosas ${ }^{5}$, o quizás se trate de síntomas no recuperados de cuadros previos más típicos o reactivaciones parciales ${ }^{7}$. Cabe destacar que aproximadamente un tercio de los pacientes que buscan tratamiento especializado no cumplen todos los criterios diagnósticos, ya sea para anorexia nerviosa o para bulimia nerviosa 5 .

Factores de riesgo. Aunque las causas de los trastornos alimentarios permanecen oscuras, la evidencia sugiere que los cambios peripuberales en la silueta corporal y en el peso, predisponen a

\section{Tabla 1. C riterios diagnósticos según el D SM -IV-T R para trastorno de la conducta alimentaria no especificado (F50.9) [307.50]*}

A. En mujeres se cumplen todos los criterios diagnósticos para la anorexia nerviosa, pero las menstruaciones son regulares

B. Se cumplen todos los criterios diagnósticos para la anorexia nerviosa excepto que, a pesar de existir una pérdida de peso significativa, el peso del individuo se encuentra dentro de los límites normales

C. Se cumplen todos los criterios diagnósticos para la bulimia nerviosa, con la excepción de que las comilonas y las conductas compensatorias inadecuadas aparecen menos de 2 veces por semana o durante menos de 3 meses

D. Empleo regular de conductas compensatorias inapropiadas después de ingerir pequeñas cantidades de comida por parte de un individuo de peso normal (por ejemplo: provocación del vómito después de haber comido dos galletas)

E. Masticar y expulsar, pero no tragar, cantidades importantes de comida

F. Trastorno compulsivo: se caracteriza por comilonas recurrentes en ausencia de la conducta compensatoria inapropiada típica de la bulimia nerviosa (ver Apéndice B del DSM-IV para los criterios que se sugieren)

*Traducida y adaptada de: American Psychiatric Association: Diagnostic and Statistical Manual of Mental Disorders. $5^{\text {th }}$ ed. Washington (DC): American Psychiatric Association, 2000. 


\section{Tabla 2. Criterios de investigación según el D SM -IV-T R (apéndice B) para el trastorno por atracones*}

A. Episodios recurrentes de comilonas. Un episodio de comilona se caracteriza por las dos condiciones siguientes:

1. Ingesta en un corto período de tiempo (por ejemplo: en 2 horas) de una cantidad de comida definitivamente superior a la que la mayoría de la gente podría consumir en el mismo tiempo y bajo circunstancias similares

2. Sensación de pérdida del control sobre la ingesta durante el episodio (por ejemplo: sensación de que uno no puede parar de comer o controlar qué o cuánto está comiendo)

B. Los episodios de comilonas se asocian a tres (o más) de los siguientes síntomas:

1. Ingesta mucho más rápida de lo normal

2. Comer hasta sentirse desagradablemente lleno

3. Ingesta de grandes cantidades de comida a pesar de no tener hambre

4. Comer a solas para esconder su voracidad

5. Sentirse a disgusto con uno mismo, depresión, o gran culpabilidad después de la comilona

C. Profundo malestar al recordar las comilonas

D. Las comilonas tienen lugar como promedio, al menos 2 días a la semana durante 6 meses

- Nota: El método para determinar la frecuencia difiere del empleado en el diagnóstico de la bulimia nerviosa; futuros trabajos de investigación aclararán si el mejor método para establecer un umbral de frecuencia debe basarse en la cuantificación de los días en que hay comilonas o en la cuantificación de su número

E. La comilona no se asocia a estrategias compensatorias inapropiadas (por ejemplo: purgas, ayuno, ejercicio excesivo) y no aparece exclusivamente en el transcurso de una anorexia nerviosa o una bulimia nerviosa

*Traducida y adaptada de: American Psychiatric Association: Diagnostic and Statistical Manual of Mental Disorders. $5^{\text {th }}$ ed. Washington (DC): American Psychiatric Association, 2000.

Tabla 3. Criterios para anorexia nerviosa atípica (F50.1) según la IC D -10*

Este término debe ser utilizado para los casos en los que faltan una o más de las características principales de la anorexia nerviosa (F50.0), como amenorrea o pérdida significativa de peso, pero que por lo demás presentan un cuadro clínico bastante característico. Este tipo de enfermos es más frecuente en psiquiatría de interconsulta y enlace y en atención primaria. También pueden incluirse aquí enfermos que tengan todos los síntomas importantes de la anorexia nerviosa, pero en grado leve. Este término no debe de utilizarse para trastornos de la conducta alimentaria que se parecen a la anorexia nerviosa pero que son debidos a una etiología somática conocida.

*Traducida y adaptada de: World Health Orzanization. The ICD-10 classification of mental and behavioural disorders: Clinical descriptions and diagnostic guidelines. Geneve: WHO, 1992.

\section{Tabla 4. C riterios para bulimia nerviosa atípica (F50.3) según la IC D -10*}

Este término debe ser utilizado para los casos en los que faltan una o más de las características principales de la bulimia nerviosa (F50.2), pero que por lo demás presentan un cuadro clínico bastante típico. Los enfermos tienen con frecuencia un peso normal o incluso superior a lo normal, pero presentan episodios repetidos de ingesta excesiva seguidos de vómitos o purgas. No son raros síndromes parciales acompañados de síntomas depresivos (si estos síntomas satisfacen las pautas de un trastorno depresivo debe hacerse un doble diagnóstico.

Incluye: Bulimia con peso normal

*Traducida y adaptada de: World Health Orzanization. The ICD-10 classification of mental and behavioural disorders: Clinical descriptions and diagnostic guidelines. Geneve: WHO, 1992. 


\title{
Tabla 5. C riterios para hiperfagia en otras alteraciones psicológicas (F50.4) según la IC D -10*
}

Ingesta excesiva como una reacción a acontecimientos estresantes y que da lugar a obesidad. Duelos, accidentes, intervenciones quirúrgicas y acontecimientos emocionalmente estresantes pueden dar lugar a una "obesidad reactiva", en especial en enfermos predispuestos a la ganancia de peso.

La obesidad como causa de alteraciones psicológicas no debe ser codificada aquí. La obesidad puede hacer que el enfermo se sienta muy sensibilizado acerca de su aspecto y desencadenar una falta de confianza en las relaciones interpersonales. Puede exagerarse la valoración subjetiva de las dimensiones corporales. Para codificar la obesidad como causa misma de alteración psicológica se deben usar categorías tales como F38.-, otros trastornos del humor (afectivos), F41.2, trastorno mixto ansioso-depresivo, o F48.9, trastorno neurótico sin especificación, más un código de E66.- para indicar el tipo de obesidad.

Incluye: Hiperfagia psicógena

Excluye: Polifagia sin especificación (R62.2)

Obesidad (E66)

*Traducida y adaptada de: World Health Orzanization. The ICD-10 classification of mental and behavioural disorders: Clinical descriptions and diagnostic guidelines. Geneve: WHO, 1992.

\section{Tabla 6. C riterios para vómitos en otras alteraciones psicológicas (F50.5) según la IC D -10*}

\author{
Además de la bulimia nerviosa en las que el vómito es autoprovocado, pueden presentarse vómitos \\ repetidos en los trastornos disociativos (de conversión) (F44.-), en la hipocondria (F45.2), en la que \\ los vómitos pueden ser uno de los múltiples síntomas corporales, y en el embarazo, donde los \\ factores emocionales pueden contribuir a la aparición de vómitos y náuseas recurrentes. \\ Incluye: Vómitos psicógenos \\ Hiperemesis gravídica psicógena \\ Excluye: Náuseas y vómitos sin especificación (R11)
}

*Traducida y adaptada de: World Health Orzanization. The ICD-10 classification of mental and behavioural disorders: Clinical descriptions and diagnostic guidelines. Geneve: WHO, 1992.

\section{Tabla 7. 0 tros trastornos de la conducta alimentaria (F50.8) según la IC D -10*}

Incluye: Disminución psicógena del apetito

Pica de origen orgánico en adultos

F50.9 Trastorno de la conducta alimentaria sin especificación

*Traducida y adaptada de: World Health Orzanization. The ICD-10 classification of mental and behavioural disorders: Clinical descriptions and diagnostic guidelines. Geneve: WHO, 1992.

las mujeres jóvenes a desarrollar actitudes alimentarias no saludables. Un desorden alimentario puede diagnosticarse en alrededor de $10 \%$ de las adolescentes con diabetes mellitus insulino-dependientes, en las cuales la ganancia ponderal, la restricción de la dieta y la preocupación por los alimentos, pueden predisponer a desarrollar una patología alimentaria clínica o subclínica ${ }^{8}$. La coexistencia de estas condiciones podría conducir a un pobre control metabólico con un riesgo elevado para complicaciones microvasculares, que requiere ser explorado por especialistas.

El aumento excesivo de actividad física en las pacientes anorécticas, al menos un año previo a 
su diagnóstico, denota que este antecedente puede ser significativo en el desarrollo del desorden, considerándose como un signo precoz de alarma en muchachas adolescentes 9 . El $11,7 \%$ de las atletas que desempeñan actividades estéticas (principalmente bailarinas) versus 5,8\% en deportistas sin esta actividad y $4,4 \%$ de los controles, han presentado un trastorno de la conducta alimentaria no especificado en algún momento de sus vidas ${ }^{10}$. Las características más comunes descritas en atletas femeninas con trastornos alimentarios subclínicos son: preocupación por los alimentos, consumo de energía, distorsión de la imagen corporal e insatisfacción con el peso corporal, influencia inapropiada de éste en la autoevaluación, intenso temor a aumentar de peso, aunque sea en el rango normal o levemente por debajo de él, uso de uno o más métodos patológicos de control ponderal, ingesta alimentaria influida por sentimientos de desprecio hacia sí misma, ausencia de trastorno médico que explique la restricción energética, pérdida de peso o mantención de peso bajo corporal y disfunción menstrual ${ }^{10}$.

Se han detectado factores comunes de riesgo para anorexia y bulimia nerviosas, trastorno por comilonas y síndromes parciales ${ }^{12}$ : género femenino, etnia caucásica, problemas gastrointestinales y alimentarios en la infancia, preocupaciones en torno al peso elevado y a la silueta, autoevaluación negativa, abuso sexual, otras experiencias adversas y en general, morbilidad psiquiátrica. Asimismo, las adolescentes inicialmente delgadas y que adhieren a los estándares perfeccionistas, pueden estar en peligro para desarrollar síndromes parciales o completos anorécticos y el afecto negativo puede contribuir al desarrollo del ciclo comilonas-purgas $^{13}$, conjuntamente con la utilización de defensas psicológicas inmaduras y neuróticas ${ }^{14}$.

Epidemiología. Son patologías alimentarias muy comunes, representando los tres cuartos de todos los casos comunitarios de desórdenes alimentarios $^{15}$ y su prevalencia fluctúa entre $2,4 \%$ y $12,6 \%$ en poblaciones adolescentes de sexo femenino ${ }^{15,16}$, cifras que superan las de los cuadros anorécticos y bulímicos completos, que oscilan entre $0,3 \%$ y $3,7 \%$ y $1 \%$ a $4,2 \%$, respectivamente 5 .

Son los trastornos alimentarios más comunes observados en los centros de tratamiento. Ciertos programas especializados han informado que $25 \%$ a $60 \%$ de los casos tratados caen en el rango no especificado $^{17,18}$. Button et $\mathrm{al}^{19}$ hallaron una prevalencia de $42,8 \%$ de trastornos de la conducta alimentaria no especificados en un centro terapéutico ad hoc, cifra similar a la de Ricca et $\mathrm{al}^{20}$ que alcanzó 43,8\%, destacando que representaron el consumo de casi la mitad de los recursos del servicio.

Los síndromes parciales o completos de anorexia nerviosa se pueden observar en los familiares de sexo femenino, tanto de pacientes anorécticas como bulímicas ${ }^{21}$. Para los síndromes parciales de anorexia nerviosa, los riesgos relativos fueron 5,6 y 6,1, y, para bulimia nerviosa fueron 2,1 y 2,2 en las parientes femeninas de las probandas anorécticas y bulímicas, respectivamente.

El trastorno por comilonas es el subgrupo no especificado más diferenciado y mejor estudiado desde la última década. Aunque actualmente no es un diagnóstico aprobado por el DSM-IV-TR ${ }^{2}$, está considerado como uno de los criterios de investigación. Consiste en alteraciones en una o más de las siguientes esferas: conductual (ej: comilonas), somática (obesidad común aunque no es requisito), y psicológica (ej: insatisfacción corporal, baja autoestima, depresión $)^{5}$.

Se estima que entre $2 \%$ y $5 \%$ de los norteamericanos sufre esta condición que afecta a ambos géneros y se difunde a lo largo de diversos grupos étnicos ${ }^{5}$. Su prevalencia es mayor en adultos que en adolescentes y frecuentemente se asocia a sobrepeso u obesidad. Los pacientes comedores compulsivos obesos muestran elevación de la presión arterial, deterioro visual, asma y otros problemas respiratorios, alteraciones cardiacas, diabetes o artritis $^{22}$. Los pacientes obesos que presentan comilonas son vulnerables a trastornos del sueño, dolores musculares crónicos cervicales y lumbares ${ }^{23}$ que interfieren significativamente en sus actividades cotidianas. Los pacientes con trastorno por comilonas están propensos a sufrir depresión mayor, desorden de pánico ${ }^{24}$ y alrededor de un tercio de las pacientes comedoras compulsivas posee un trastorno de personalidad limítrofe $^{25}$.

Características clínicas. Es conveniente, para comprender las manifestaciones sintomatológicas 
de los desórdenes subclínicos, primeramente conocer la anorexia nerviosa y la bulimia nerviosa propiamente tales. Parte de los cuadros clasificados como trastornos atípicos son, en realidad, una anorexia o una bulimia que se detectan en fases muy iniciales, tras un corto periodo de duración de entre cinco u ocho meses. Lo positivo a este respecto es que el trastorno se pesquisa a tiempo, cuando aún no se ha desarrollado de forma completa. Así, los pacientes con una anorexia incipiente tienen mejor pronóstico, se suelen recuperar con normalidad y recuperar peso.

Las pacientes que asisten a unidades de trastornos alimentarios, con cuadros no especificados y aquellas con desórdenes típicos poseen atributos psicopatológicos similares, tanto anorécticos como bulímicos ${ }^{20,26}$.

Ricca et $\mathrm{al}^{20}$ y Keel ${ }^{26}$ subrayan que aunque aparentemente podrían catalogarse de "parciales" o "subclínicos", son patologías serias que implican un compromiso físico y mental. La baja densidad ósea no solamente ocurre en las mujeres que reunen los requisitos para el desorden completo, sino además en aquellas que satisfacen los criterios, excepto la instalación de amenorrea, evidenciando adicionalmente altos y comparables niveles de distorsión de la imagen corporal ${ }^{5}$. Por otro lado, una paciente que presenta comilonas una vez a la semana pero cumple todos los criterios para bulimia nerviosa, también muestra una vulnerabilidad significativa para desarrollar trastornos afectivos y de ansiedad. El masticar y escupir los alimentos son síntomas frecuentes en todos los grupos de trastornos alimentarios, presente aproximadamente en $22 \%$ de los casos. Kovacs et $\mathrm{al}^{27}$ determinaron que aquéllos con anorexia nerviosa y trastornos de la conducta alimentaria no especificados que los exhibían, mostraron una patología alimentaria más severa, no así para la bulimia nerviosa, que informaron sólo una mayor distorsión de la imagen corporal. El trastorno por comilonas no se relacionó con estas manifestaciones.

En las afecciones subclínicas se han descrito similares comorbilidades que en los trastornos completos. En un estudio comunitario efectuado en mujeres adultas jóvenes, las conductas severas de comilonas se asociaron significativamente con el uso de alcohol y las restrictivas y purgativas con el de estimulantes/anfetaminas y abuso de somníferos ${ }^{28}$.
Curso y pronóstico. Los trastornos de la conducta alimentaria no especificados cubren un amplio espectro, por lo que es difícil generalizar comentarios sobre el curso y pronóstico del grupo total. Además, temporalmente, ocurren cambios considerables desde una a otra categoría diagnóstica dentro de los trastornos alimentarios -incluyendo los no especificados ${ }^{4}-$ o en el futuro, éstos pueden reunir todos los criterios diagnósticos para anorexia o bulimia ${ }^{29}$, pudiendo asociarse con la misma comorbilidad, curso y mejoría, como si reuniesen los requisitos para el cuadro completo e inclusive pueden ser potencialmente mortales ${ }^{18,30}$.

Es primordial realizar una evaluación clínica restrospectiva de posibles expresiones sugerentes de estos desórdenes en el análisis longitudinal de estos casos, las que a veces aparecen con varios años de antelación. Generalmente son muchachas adolescentes que sufren problemas físicos y psicológicos que varían dentro de un vasto rango de severidad, debido a la comorbilidad o como consecuencia de sus patrones alimentarios: constipación crónica, síntomas dispépticos, náuseas, dolor abdominal, fatiga, cefaleas, hipotensión, disfunción menstrual, distimia, trastornos depresivos y ansiosos o mal uso y abuso de sustancias ${ }^{29}$. En contraste con aquellas no afectadas, las adolescentes con trastornos parciales están en alto riesgo para desarrollar un trastorno completo, aunque, muchas pueden tener una remisión espontánea ${ }^{29}$. No obstante, comúnmente realizan más dieta en el año previo, informan un mayor cambio en la cantidad de ingesta alimentaria en situaciones de estrés y demuestran una elevada psicopatología general ${ }^{31}$.

De esta manera, estos cuadros pueden ser tan peligrosos o amenazantes como la anorexia o la bulimia nerviosas completas, su clasificación de no especificados en este sentido, es un mero tecnicismo. La restricción calórica, las purgas, las comilonas, el ejercicio excesivo desplegado con regularidad, son deteriorantes para la salud y generan gran ansiedad; aunque habitualmente comienzan de manera gradual, eventualmente pueden derivar en cuadros más graves, más difíciles de controlar y más complejos de tratar ${ }^{1}$.

Otros tipos clínicos. Otras patologías alimentarias, menos comunes que han sido clasificadas como no especificados son: 
- Pica: ingestión persistente de sustancias no nutritivas (ej: tierra, metal, papel, etc.) inapropiada para el nivel de desarrollo, durante un periodo de al menos un mes $^{2}$.

- Síndrome de Prader-Willi: trastorno congénito caracterizado por hipotonía, baja estatura, extremidades pequeñas, hipogonadismo y diversos grados de incapacidad mental, episodios de hiperfagia con apetito insaciable, trastornos del sueño, conducta agresiva, obesidad, diabetes tipo II, retardo en el desarrollo psicosexual, deterioro del lenguaje y problemas dentales ${ }^{32}$.

- Síndrome del comer nocturno: caracterizado por anorexia matinal, hiperfagia vespertina e insomnio, con despertares seguidos por consumo nocturno de alimentos ${ }^{33}$.

- Ortorexia, obsesión de comer alimentos considerados saludables por la persona, lo que incluso puede llevar a una desnutrición severa y a la muerte. Típicamente se evitan las grasas, preservantes o productos animales ${ }^{34}$.

- Vigorexia (trastorno dismórfico muscular o anorexia inversa): preocupación obsesiva por el físico y distorsión del esquema corporal, acompañados de una adicción a la actividad física con el fin de lograr un desarrollo muscular mayor de lo normal, ya que los pacientes, comúnmente de sexo masculino, se sienten débiles y pequeños. Es común entre los fisicoculturistas, cuyas dietas extremadamente proteico-calóricas y el abuso de esteroides, los lleva a una insuficiencia renal y a otros trastornos somáticos, psicopatológicos y sociales ${ }^{35}$.

Síntomas y signos de alarma. Si bien no todas las personas desarrollarán un trastorno alimentario, corrientemente los cuadros parciales comienzan con una dieta que siguen para recuperar autoestima y control, generando señales que requieren especial vigilancia: fluctuaciones en la historia ponderal, preocupación sobrevalorada del peso y silueta corporal en la evaluación personal, oscilaciones anímicas, rutinas rígidas de alimentación, aumento en las horas del ejercicio físico y tiempo invertido en la planificación de la alimentación. Se debe colocar atención a los síntomas y signos de alarma señalados en Tablas 8 y 9 .

\section{APROXIMACIONES TERAPÉUTICAS}

Los trastornos de la conducta alimentaria no especificados ameritan un tratamiento similar a los cuadros completos 5 y no deben considerarse como afecciones triviales ${ }^{20}$. La importancia del diagnóstico y la intervención precoces, nunca será enfatizada suficientemente, ya que mejora ostensiblemente el pronóstico ${ }^{29}$. Comúnmente tardan en diagnosticarse porque al ser síndromes parciales repercuten menos en la vida de las personas y disminuye su motivación a solicitar ayuda profesional, pudiendo ser prevenidos, mediante intervenciones a nivel primario cognitivo-conductuales a través de Inter-

Tabla 8. Síntomas y signos físicos de alarma*

Pérdida de peso de origen desconocido en jóvenes, sobre todo de sexo femenino

Falla en el crecimiento normal para la edad y peso del paciente

Irregularidades de la menstruación o amenorrea

Se aprecia debilidad. En período de exámenes, por ejemplo, llegan a producirse desmayos por no

haber desayunado

Complicaciones médicas por ejercicio físico abusivo

Osteoporosis en jóvenes

Hirsutismo o lanugo

Anomalías dentarias

Hipertrofia parotídea

Callosidades en los nudillos de las manos

Constante sensación de frío

*Tomada de: Behar R. Trastornos de la conducta alimentaria: Clínica y epidemiología. En: Behar R, Figueroa G, editores. Anorexia nerviosa y bulimia. Clínica y terapéutica. Santiago de Chile: Editorial Mediterráneo, 2004; 17-54. 


\section{Tabla 9. Síntomas y signos conductuales de alarma*}

Comer en solitario, desaparecer inmediatamente después de las comidas y encerrarse en el baño Preocupación excesiva por la composición calórica de los alimentos y por la preparación de los alimentos

Miedo exagerado al sobrepeso o a la ganancia ponderal

Creciente interés en temas gastronómicos, ropa y modas

Valoración del peso o figura como prioridad. Preocupación exagerada por la imagen (pesaje frecuente) y mala imagen corporal (rechazo hacia ella). Uso exclusivo de pantalones y tendencia a ocultar ciertas partes del cuerpo

Evitación de alguna comida o alimento de forma frecuente

Retraimiento de amistades y compañeros

Aumento de actividades "útiles" y horas de estudio

Miembro de un subgrupo vulnerable de población (bailarinas, modelos, gimnastas, actrices, etc.)

Períodos de semiayuno alternados con ingesta normal

Mantenimiento crónico de dietas hipocalóricas (tres o más dietas de adelgazamiento el último año), en especial en ausencia de obesidad o sobrepeso

Humor deprimido a irritable

Pérdida de interés o placer frente a las actividades cotidianas

Hiperactividad

*Tomada de: Behar R. Trastornos de la conducta alimentaria: Clínica y epidemiología. En: Behar R, Figueroa G, editores. Anorexia nerviosa y bulimia. Clínica y terapéutica. Santiago de Chile: Editorial Mediterráneo, 2004; 17-54.

net, reduciendo significativamente la preocupación por el peso y figura en grupos de riesgo ${ }^{36}$.

Se requiere un tratamiento individualizado comprensivo, interdisciplinario, orientado a las múltiples dimensiones etiopatogénicas de estas afecciones, que provea un meticuloso seguimiento ${ }^{5}$ para lograr una sólida recuperación y prevención de recaídas, con aproximaciones análogas a las de los cuadros completos o más típicos, aunque algo más mesurados, con objetivos crecientes y prudentes para evitar resistencias ${ }^{6}$. Estas estrategias en lo posible deberían ser ambulatorias, encaminadas a las correcciones somáticas, con abordajes psicoeducativos sobre la ingesta, ejercicios, valoración del cuerpo, naturaleza de la enfermedad y psicofármacos; cognitivo-conductuales, de apoyo, directivas, dirigidas a mejorar la obsesividad, el ánimo, la autoestima, la identidad, las relaciones, los intereses, a evitar las manipulaciones y al desarrollo de defensas psicológicas alternativas ${ }^{6}$.

IMPLICANCIAS

Andersen et $\mathrm{al}^{37}$ estiman que la contundente cantidad de casos atípicos, sugiere que los diag- nósticos actuales de anorexia y de bulimia son demasiado limitados, definidos por criterios basados en la investigación, o que son necesarias las jerarquías adicionales, que en estos momentos se catalogan por exclusión en los no especificados. Por otra parte, tal diagnóstico conduce a desorientación e incertidumbre en relación a los métodos terapéuticos a utilizar, además que en ciertos países las compañías de seguros no los cubren, ya que consideran que no corresponden a verdaderos trastornos alimentarios ${ }^{37}$.

Franko y Omori ${ }^{38}$ apoyan la hipótesis de la continuidad en las afecciones alimentarias, desde la emergencia de las preocupaciones en torno al peso y silueta, pasando por síndromes parciales, hasta la anorexia y bulimia nerviosas completas, mientras que Andersen et $\mathrm{al}^{37}$ hipotetizan que los no especificados pertenecen como subgrupos, a las categorías actuales de anorexia y bulimia, ya que reúnen los rasgos esenciales y críticos de estas entidades nosológicas. Rockert et al ${ }^{39}$ dividen los no especificados en seis categorías, sugiriendo que alguna porción de éstos se parece a la anorexia o a la bulimia nerviosas sindromáticamente completas y el resto aparece como 
heterogéneo. Fairburn et $\mathrm{al}^{4}$ proponen al respecto el concepto de "trastornos alimentarios mixtos".

Garfinkel et $\mathrm{al}^{40}$ cuestionan la amenorrea como criterio diagnóstico esencial para la anorexia, debido a que algunas mujeres pierden su menstruación precozmente después de comenzar su pérdida de peso, o incluso antes, mientras que otras continúan con sus reglas todavía después de una sustancial pérdida ponderal. Lo que sí es un requerimiento nuclear para la homogeneidad diagnóstica, es la presencia de temor mórbido a engordar o la implacable motivación por la delgadez, bien conceptualizada como "creencia sobrevalorada" por Andersen ${ }^{37}$.

El Grupo de Trabajo sobre Trastornos Alimentarios de la Asociación Psiquiátrica Americana ${ }^{5}$ plantea la necesidad de una investigación sistematizada de estos problemas. Rockert et al ${ }^{39}$ sugieren que el énfasis debería incluir la exploración de las diferencias de personalidad entre individuos con anorexia nerviosa, bulimia nerviosa y no especificados, así como el análisis de los diversos desenlaces terapéuticos para los distintos grupos.

Para Chinchilla ${ }^{7}$, los trastornos parciales alimentarios son cuadros muy heterogéneos que necesitan una nueva reconsideración nosológico-

\section{REFERENCIAS}

1. Sloan D, Mizes J, Epstein E. Empirical classification of eating disorders. Eat Behav 2005; 6: 53-62.

2. American Psychiatric Association. Diagnostic and statistical manual of mental disorders. Text Revision. Washington (DC): American Psychiatric Association, 2000 .

3. World Health Organization. The ICD-10 classification of mental and behavioural disorders: Clinical descriptions and diagnostic guidelines. Geneve: WHO, 1992.

4. Fairburn C, BOHN K. Eating disorder NOS (EDNOS): An example of the troublesome "not otherwise specified" (NOS) category in DSM-IV. Behav Res Ther 2005; 43: 691-701.

5. American Psychiatric Association. Practice guideline for the treatment of patients with eating disorders. Third edition. American Psychiatric Association Work Group on Eating Disorders. Am J Psychiatry 2006; 58-60.

6. Rusch M, ANDRIS D. Maladaptive eating patterns after weight-loss surgery. Nutrition in Clinical Practice 2007; 22: 41-9. conceptual. El interés radicaría en el diagnóstico temprano, donde posiblemente se encuentre menos gravedad, más atipicidad, menor comorbilidad general, mejor estado de personalidad y posiblemente posean una multifactoriedad causal incompleta o más leve.

El principal propósito de la puesta al día en esta temática, cada vez más vigente y que afecta a jóvenes en plena edad productiva, es transmitir la información actualizada de estos síndromes alimentarios tan variados, sutiles y camaleónicos, factibles de pesquisar en cualquier entrevista y examen físico habitual, tanto en un contexto médico-quirúrgico general, como de especialidades o subespecialidades médicas, idealmente con el fin de efectuar su prematuro reconocimiento y manejo, $\mathrm{y}$, de esta manera abortar su cronificación en el tiempo e impedir su transformación en patologías completas, con todas las consecuencias que ello implica. El rol del médico general o familiar debe focalizarse tanto en la prevención primaria, mediante una temprana psicoeducación, como en el establecimiento de una alianza terapéutica dinámica y consistente, al enlace con el equipo especialista y al seguimiento a largo plazo posterior al alta, que requieren indispensablemente estas afecciones.

7. Chinchilla A. Trastornos de la conducta alimentaria. Anorexia y bulimia nerviosas, obesidad y atracones. Madrid: Masson, 2003.

8. Verrotti A, Catino M, De luca F, Morgese G, Chiarelli F. Eating disorders in adolescents with type 1 diabetes mellitus. Acta Diabetol 1999; 36: 21-5.

9. Davis C, Blackmore E, Katzman D, Fox J. Female adolescents with anorexia nervosa and their parents: A case-control study of exercise attitudes and behaviours. Psychol Med 2005; 35: 377-86.

10. Bachner-Melman R, Zohar A, Ebstein R, Elizur Y, Constantini N. How anorexic-like are the symptom and personality profiles of aesthetic athletes? Med Sci Sports Exerc 2006; 38: 628-36.

11. Beals K, Manore M. Behavioral, psychological, and physical characteristics of female athletes with subclinical eating disorders. Int J Sport Nutr Exerc Metab 2000; 10: 128-43.

12. Jacobi C, Hayward C, De Zwann M, Kraemer H, Agras W. Coming to terms with risk factors for eating disorders: Application of risk terminology and suggestions for a general taxonomy. Psychol Bull 2004; 130: 19-65. 
13. Tyrka A, Waldron I, Graber J, Brooks-Gunn J. Prospective predictors of the onset of anorexic and bulimic syndromes. Int J Eat Disord 2002; 32: 282-90.

14. Stein D, Bronstein Y, Weizman A. Defense mechanis$\mathrm{ms}$ in a community-based sample of female adolescents with partial eating disorders. Int J Psychiatry Med 2003; 33: 343-55.

15. Machado P, Machado B, Gonçalves S, Hoek H. The prevalence of eating disorders not otherwise specified. Int J Eat Disord 2007; 40: 212-7.

16. Zini A, Siani R, Sandri M, Soardo F, Siciliani O. Partial syndromes in eating disorders: A prevalence study on a sample of Italian adolescents. Eat Weight Disord 2007; 12: 125-31.

17. Mizes J, SloAN D. An empirical analysis of eating disorder, not otherwise specified: Preliminary support for a distinct subgroup. Int J Eat Disord 1998; 23: 233-42.

18. Williamson D, Gleaves D, Suzanne S, Savin S. Empirical classification of eating disorder not otherwise specified: Support for DSM-IV changes. J Psychopathology Behav Assessment 1992; 14: 201-16.

19. Button E, Benson E, Nollett C, Palmer R. Don't forget EDNOS (eating disorder not otherwise specified): Patterns of service use in an eating disorders service. Psychiatric Bulletin 2005; 29: 134-6.

20. Ricca V, Mannucci E, Mezzani B, Di Bernardo M, Zucchi T, Paionni A et al. Psychopathological and clinical features of outpatients with an eating disorder not otherwise specified. Eat Weight Disord 2001; 6: 157-65.

21. Strober M, Freeman R, Lampert C, Diamond J, Kaye W. Controlled family study of anorexia nervosa and bulimia nervosa: Evidence of shared liability and transmission of partial syndromes. Am J Psychiatry 2000; 157: 393-401.

22. Bulik C, Sullivan P, Kendler K. Genetic and environmental contributions to obesity and binge eating. Int J Eat Disord 2003; 33: 293-8.

23. Reichborn-Kjennerud T, Bulik C, Sullivan P, Tambs K, Harris J. Psychiatric and medical symptoms associated with broadly defined binge-eating disorder. In: Bulik C, Reichborn-Kjennerud T, editors. Medical morbidity in binge eating disorder. Int J Eat Disord 2003; 34 Suppl: 39-46.

24. Wilfley D, Wilson G, Agras W. The clinical significance of binge eating disorder. IntJ Eat Disord 2003; 34 Suppl: 96-106.

25. Marino M, Zanarini M. Relationship between EDNOS and its subtypes and borderline personality disorder. Int J Eat Disord 2001; 29: 349-53.
26. Keel P. Purging disorder: Subthreshold variant or full-threshold eating disorder? Int J Eat Disord 2007; 40 Suppl: 89-94.

27. Kovacs D, Mahon J, Palmer R. Chewing and spitting out food among eating-disordered patients. Int J Eat Disord 2002; 32: 112-5.

28. Piran N, Robinson S. The association between disordered eating and substance use and abuse in women: A community-based investigation. Women Health 2006; 44: 1-20.

29. Chamay-Weber C, Narring F, Michaud P. Partial eating disorders among adolescents: A review. J Adolesc Health 2005; 37: 417-27.

30. Le Grange D, Binford R, Peterson C, Crow S, Crosby R, KLEIN M ET AL. DSM-IV threshold versus subthreshold bulimia nervosa. Int J Eat Disord 2006; 39: 462-7.

31. Stein D, Luria O, Tarrasch R, Yoeli N, Glick D, Elizur A Eт AL. Partial eating disorders in newly drafted female recruits in Israel. Arch Women's Mental Health 1999; 2: 107-15.

32. Behar R. Trastornos de la conducta alimentaria: Clínica y epidemiología. En: Behar R, Figueroa G, editores. Anorexia nerviosa y bulimia. Clínica y terapéutica. Santiago de Chile: Editorial Mediterráneo, 2004; 17-54.

33. Stunkard A, Grace W, Wolff H. The night-eating syndrome: A pattern of food intake among certain obese patients. Am J Med 1955; 19: 78-86.

34. Bratman S, Knight D. Health food junkies. New York: Broadway Books, 2000.

35. Baile J. Vigorexia ¿cómo reconocerla y evitarla? Madrid: Editorial Síntesis, 2005.

36. Taylor C, Bryson S, Luce K, Cunning D, Doyle A, Abascal L et al. Prevention of eating disorders in atrisk college-age women. Arch Gen Psychiatry 2006; 63: 881-8.

37. Andersen A, Bowers W, Watson T. A slimming program for eating disorders not otherwise specified. Reconceptualizing a confusing, residual diagnostic category. Psychiatr Clin North Am 2001; 24: 271-80.

38. Franko D, Omori M. Subclinical eating disorders in adolescent women: A test of the continuity hypothesis and its psychological correlates. J Adolesc 1999; 22: 389-96.

39. Rockert W, Kaplan A, Olmsted M. Eating disorder not otherwise specified: The view from a tertiary care center. Int J Eat Disord 2007; 40: 99-103.

40. Garfinkel P, Lin E, Goering P, Spegg C, Goldbloom D, KENNEDY $S$ ET AL. Should amenorrhea be necessary for the diagnosis of anorexia nervosa? Evidence from a Canadian community sample. Br J Psychiatry 1996; 168: 500-6. 